\title{
Nailfold capillaroscopy as a risk factor for pulmonary arterial hypertension in systemic lupus erythematosus patients
}

Juliana Fernandes Sarmento Donnarumma ${ }^{1}$, Eloara Vieira Machado Ferreira ${ }^{2}$, Jaquelina Ota-Arakaki ${ }^{2}$ and Cristiane Kayser ${ }^{1,3^{*}}$ (i)

\begin{abstract}
Background: Pulmonary arterial hypertension $(\mathrm{PAH})$ is a rare and severe complication of systemic lupus erythematosus (SLE). This study aimed to evaluate clinical and laboratory risk factors associated with PAH in SLE patients.

Methods: This was a retrospective case-control study in which patients with SLE with PAH (SLE-PAH) confirmed by right heart catheterization (RHC) were compared with SLE patients without PAH. Clinical and demographic variables related to SLE and PAH and nailfold capillaroscopy were evaluated by reviewing the medical records of the patients.

Results: Twenty-one patients with SLE-PAH and 44 patients with SLE without PAH matched for sex and disease duration were included. The scleroderma (SD) pattern on nailfold capillaroscopy was more frequently found in patients with SLE-PAH than in those without PAH (56.3\% versus $15.9 \%$, respectively, $p=0.002)$. By univariate analysis, Raynaud's phenomenon, history of abortion, and SD pattern on capillaroscopy were associated with PAH. Arthritis was a protective factor for PAH development. Multivariate analysis showed that the SD pattern on capillaroscopy was the only variable associated with a significantly higher risk of $\mathrm{PAH}$, with an odds ratio of 6.393 (95\% confidence interval, 1.530-26.716; $p=0.011$ ).

Conclusion: In this study, SD pattern was associated with a 6.3-fold increased risk for PAH development in SLE patients, suggesting that nailfold capillaroscopy might be useful as a screening method to identify SLE patients with a high risk of developing this severe complication.
\end{abstract}

Keywords: Systemic lupus erythematosus, Pulmonary arterial hypertension, Risk factors, Nailfold capillaroscopy

\section{Background}

Systemic lupus erythematosus (SLE) is a rare chronic autoimmune disease of multifactorial etiology. Symptoms are heterogeneous and vary from mild to severe and potentially fatal systemic manifestations [1].

Pulmonary hypertension (PH) is a complex condition defined as an elevation of the mean pulmonary artery pressure (MPAP) ( $\geq 25 \mathrm{mmHg}$ ) at rest on right heart catheterization (RHC). The term pulmonary arterial

\footnotetext{
*Correspondence: criskayser@terra.com.br

'Rheumatology Division, Medicine Department, Escola Paulista de Medicina, Universidade Federal de São Paulo (UNIFESP), São Paulo, SP, Brazil

${ }^{3}$ Disciplina de Reumatologia da Universidade Federal de São Paulo, Rua

Botucatu 740, $3^{\circ}$ andar, São Paulo, SP 04023-062, Brazil

Full list of author information is available at the end of the article
}

hypertension (PAH) is used to designate a group of patients with pulmonary hypertension hemodynamically defined by precapillary $\mathrm{PH}$, with pulmonary capillary wedge pressure (PCWP) $\leq 15 \mathrm{mmHg}$ and an increase in the pulmonary vascular resistance (PVR) $[2,3]$.

$\mathrm{PAH}$ associated with autoimmune rheumatic diseases represent $30 \%$ of all adult PAH cases [4-6]. Among the rheumatic diseases associated with $\mathrm{PAH}$, systemic sclerosis (SSc) and SLE are the most common $[7,8]$. The prevalence of PAH among SLE patients varies from 0.5 to $14 \%$ according to the analyzed population [9-13]. Such wide variation is mainly attributed to the diagnostic methods used. Indeed, many studies were based on estimates of the right ventricular systolic pressure

(C) The Author(s). 2019 Open Access This article is distributed under the terms of the Creative Commons Attribution 4.0 International License (http://creativecommons.org/licenses/by/4.0/), which permits unrestricted use, distribution, and 
measured on transthoracic echocardiogram, using a variety of cutoff points ( $>30$ to $45 \mathrm{mmHg}$ ) to define $\mathrm{PAH}$. Moreover, although $\mathrm{PAH}$ is the most frequent etiology of $\mathrm{PH}$, it can be associated with other aetiologies, including pulmonary veno-occlusive disease, and pulmonary fibrosis and the differential diagnosis is not always simple [7].

Among patients with SLE, PAH is associated with high morbidity and mortality [14, 15]. Despite advances in the treatment of PAH in recent decades, patients with associated autoimmune rheumatic diseases exhibit a more severe form of disease and a poorer prognosis than do patients with other forms of PAH $[8,16]$. According to reports in the literature, the 1- and 3-year survival rates for patients with SLE and PAH are 78 and $74 \%$, respectively [17]. However, in a recent cohort study of 2967 patients with PAH at various American centers, the 1-year survival rate for patients with SLE was $94 \%[8]$.

Several risk factors have been associated with the development of PAH among SLE patients, including the presence of Raynaud's phenomenon (RP), anti-ribonucleoprotein (anti-RNP) and anti-cardiolipin antibodies, digital vasculitis, livedo reticularis, serositis, and high serum endothelin-1 levels $[14,16,18-20]$. However, most studies employed echocardiography for the diagnosis of $\mathrm{PAH}$, which is a substantial limitation given its low accuracy.

Nailfold capillaroscopy (NC) is a noninvasive method that is widely used in the investigation of patients with RP. Patients with RP secondary to SSc and SSc spectrum disorders exhibit a classic microangiopathy pattern known as the scleroderma (SD) pattern, which is characterized by loss of capillary loops, capillary dilatation, and disorganization of the capillary structure [21]. Some studies of patients with SSc have found an association between $\mathrm{NC}$ abnormalities and the presence and severity of PAH, which suggests that changes in the microcirculation might play a role in the development or pathogenesis of PAH [22-25].

The aim of the present study was to identify risk factors for the development of PAH among SLE patients by analyzing clinical aspects and complementary tests, with an emphasis on $\mathrm{NC}$ as a possible method for the identification of patients with SLE and PAH.

\section{Materials and methods}

\section{Patients and study design}

In the present single-center, retrospective, case-control study conducted from 2013 to 2015, risk factors for PAH were investigated among SLE patients who developed PAH (SLE-PAH) or who did not (SLE-nPAH). Both groups were matched for sex and duration of disease (up to the outcome in group SLE-PAH) at a 2:1 ratio.
For the SLE-PAH group, the diagnosis of PAH met established criteria, especially confirmation on RHC [2]. Patients were identified through a review of medical records and via the database of $\mathrm{PH}$ patients of the Pulmonary Circulation Service, of the Pneumology Department of the Federal University of São Paulo.

Patients with SLE and without known diagnosed PAH (SLE-nPAH) were randomly selected from the SLE outpatient clinic, and those with SLE-PAH were also followed-up at the pulmonary outpatient clinic at the Federal University of São Paulo Medical School Hospital. The inclusion criteria were as follows: age of 18 years old or older; fulfilling the American College of Rheumatology (ACR) 1997 revised criteria for the classification of SLE [26]; and availability of clinical and laboratory data in the medical records at the time of inclusion. Patients with SSc overlap syndrome, rheumatoid arthritis, or polymyositis/dermatomyositis were excluded. The study was approved by the local research ethics committee (no. 503,630).

Thirty patients with PAH were identified. Five of them had not performed RHC for diagnosis and thus did not meet the inclusion criteria. Four patients had missing data in their medical records and had been subjected to one single outpatient evaluation; therefore, they could not be included in the study. As a result, the study sample comprised 21 SLE-PAH and 44 SLE-nPAH patients.

\section{Right heart catheterization}

RHC was indicated for patients with clinical suspicion of PAH and pulmonary artery pressure (PAP) $\geq 35 \mathrm{mmHg}$ on Doppler echocardiogram. PAH was defined as MPAP $\geq 25 \mathrm{mmHg}$ at rest, PCWP $\leq 15 \mathrm{mmHg}$, and an elevated PVR (> 3 Wood units-WU). The following parameters were analyzed on $\mathrm{RHC}$ at the time of $\mathrm{PAH}$ diagnosis: MPAP, cardiac index (CI), PCWP, and PVR index (PVRI). All exams were performed at the hemodynamics service of São Paulo Medical School Hospital. Interstitial lung disease, chronic thromboembolic pulmonary hypertension, congenital heart disease, significant valvular heart disease, chronic obstructive pulmonary disease, portal hypertension, schistosomiasis, immunodeficiency virus, and thyroid disorders had been previously ruled out.

\section{Clinical and laboratory data}

All the analyzed data were extracted from the patients' medical records. Attention was paid to the following variables: age, sex, ACR classification criteria for SLE, time since diagnosis of SLE and PAH, smoking, obstetric history, presence of RP and livedo reticularis, laboratory data, NC data, thrombotic events, and deaths in the SLE-PAH group. SLE activity was analyzed for all patients based on their score on the Systemic Lupus Erythematosus Disease Activity Index (SLEDAI) [27]. 
Laboratory tests included the detection of antinuclear (ANA) and anti-dsDNA antibodies by indirect immunofluorescence using HEp-2 cells and Crithidia luciliae as the substrate, respectively. Anti-Sm, anti-RNP, anti-Ro, and anti-La were investigated based on double immunodiffusion. Complement fractions (C2 and $\mathrm{C} 100)$ were measured with radial immune hemolysis. Anti-cardiolipin antibodies (anti-aCL IgG and IgM) were detected using an enzyme-linked immunosorbent assay (ELISA). Serum creatinine levels, complete blood counts, C-reactive protein (CRP) levels, and 24-h urine protein levels, were also evaluated in all subjects.

NC was performed with a stereomicroscope (SZ40, Olympus) at $10-25 \mathrm{x}$ magnification. The NC parameters considered in the analysis were as follows: $\mathrm{SD}$, normal or unspecific pattern, number of loops per $\mathrm{mm}$, avascular score, number of giant and enlarged capillaries, and microhemorrhages [28]. The avascular score was determined semiquantitatively on a scale from 0 to 3 as previously described [29]. The mean score for each parameter was calculated from the analysis of all fingers except the thumbs.

Patients with PAH were also evaluated based on the New York Heart Association (NYHA) functional classification [30], distance walked in the 6-min walk test (6MWT), forced vital capacity (FVC) and carbon monoxide diffusing capacity (Dco) on pulmonary function tests, and echocardiogram at the time of PAH diagnosis. The echocardiogram parameters considered were as follows: PAP, right ventricular size, tricuspid regurgitation velocity (TRV), and presence of pericardial effusion.

Clinical and demographic data were collected at the time of diagnosis. The data on SLEDAI and current or previous SLE treatment were obtained at the time of inclusion in the study. For the SLE-PAH group, clinical and laboratory data on $\mathrm{PAH}$ were collected at the time of $\mathrm{PAH}$ diagnosis.

\section{Statistical analysis}

The data are presented as the mean and standard deviation and as absolute and relative frequencies in the case of categorical variables. The Shapiro-Wilk test was used to investigate the normality of the variable distribution. Continuous variables were compared between groups using Student's t-test or the Mann-Whitney test. Categorical data were analyzed using the chi-square or Fisher's exact test. The significance level was set at $p<$ 0.05. To identify risk factors in the SLEP-PAH group, uni- and multivariate logistic regression analyses were performed, and the odds ratio (OR) for each factor was calculated with the corresponding 95\% confidence interval (95\% CI) relative to the SLE-nPAH group. All variables with $p<0.10$ in the univariate analysis were included in the multivariate analysis. Statistical analyses were performed using SPSS software for Windows version 19.0 (Chicago, IL).

\section{Results}

The clinical, demographic, and laboratory characteristics of the 21 patients with SLE-PAH and the 44 SLE-nPAH patients are described in Table 1 . The mean age at the time of SLE onset was 28.8 and 31.2 years in the SLE-PAH and SLE-nPAH groups, respectively. Female patients predominated in both groups. The mean duration of SLE at the time of PAH diagnosis was $6.7 \pm 6.4$ years in the SLE-PAH group. Regarding the ACR criteria, arthritis and photosensitivity were more frequent in the SLE-nPAH group (93.2 and 79.5\%, respectively) than in the SLE-PAH group (70 and 55\%, respectively). The SLEDAI score was not significant different between the groups; $29 \%$ of the patients in the SLE-nPAH group and $19 \%$ of those in the SLE-PAH group exhibited active disease (SLEDAI $\geq 4$ ). Forty-two patients $(64.6 \%)$ exhibited RP, with a trend toward a higher frequency in the SLE-PAH group than in the SLE-nPAH group (81\% versus $56.8 \%$, respectively; $p=0.057$ ).

Forty patients $(61.5 \%)$ had been pregnant at least once, without a significant difference between the groups. Only one patient in the SLE-PAH group had become pregnant after the diagnosis of $\mathrm{PAH}$, and the outcome was a preterm birth. Ten patients (15.4\%) had at least one miscarriage, with a significantly higher frequency in the SLE-PAH group than in the SLE-nPAH group (28.6\% versus 9.1\%, respectively; $p=0.042$ ) (Table 1 ). Secondary antiphospholipid syndrome was diagnosed in two patients in the SLE-PAH group and in five patients in the SLE-nPAH group.

Nailfold capillaroscopy was performed in 16 patients in the SLE-PAH group and 44 patients in the SLE-nPAH group. The SD pattern was detected in $56.3 \%$ of the SLE-PAH patients. Most patients (84.1\%) in the SLE-nPAH group exhibited a normal or unspecific pattern on capillaroscopy, and only $15.9 \%$ exhibited the SD pattern $(p=0.002)$. The number of dilated capillaries and the avascular score were significantly higher in the SLE-PAH group ( $p=0.027$ and $p=0.010$, respectively) (Table 2).

The mean RHC findings for the SLE-PAH group were as follows: MPAP $48.9 \pm 11.7 \mathrm{mmHg}, \mathrm{CI} 2.47 \pm 0.7 \quad \mathrm{~L} /$ $\mathrm{min} / \mathrm{m}^{2}$, and PVRI $10.9 \pm 8.8 \mathrm{WU} / \mathrm{m}^{2}$ (Table 3). The most frequent functional class (NYHA) at the time of diagnosis was functional class III (47.6\%), followed by class II (33.3\%); two patients (9.5\%) were categorized as class I and another two (9.5\%) as class IV. The mean distance walked in the $6 \mathrm{MWT}$ was $416.6 \pm 80.6 \mathrm{~m}$. The mean FVC was $79.6 \pm 15.2 \%$, and the mean Dco was $47.4 \pm 18.9 \%$. Regarding the echocardiography parameters, the mean PAP was $63.1 \pm 14.1 \mathrm{mmHg}$, the mean TRV was $3.71 \pm 0.41 \mathrm{~m} / \mathrm{s}$, and the mean right ventricular 
Table 1 Clinical and demographic characteristics of patients with SLE associated with PAH (SLE-PAH) or not associated with PAH (SLE-nPAH)

\begin{tabular}{|c|c|c|c|}
\hline & SLE-nPAH $n=44$ & SLE-PAH $n=21$ & $P$-value \\
\hline Age at SLE diagnosis (years) & $31.2 \pm 12.3$ & $28.8 \pm 10.2$ & 0.411 \\
\hline Age at PAH diagnosis (years) & - & $35.5 \pm 11.8$ & - \\
\hline Duration of disease at recruitment (years) & $5.6 \pm 3.2$ & $6.7 \pm 6.4$ & 0.437 \\
\hline Female sex, n (\%) & $42(95.5)$ & $20(95.2)$ & 0.969 \\
\hline Smoker, n (\%) & $0(0)$ & $1(4.8)$ & 0.145 \\
\hline Ex-smoker, n (\%) & $4(9.1)$ & $1(4.8)$ & 0.295 \\
\hline \multicolumn{4}{|l|}{ ACR SLE criteria, n (\%) } \\
\hline Malar rash & $34(77.3)$ & $13(65)$ & 0.303 \\
\hline Photosensitivity & $35(79.5)$ & $11(55)$ & 0.043 \\
\hline Discoid rash & $3(6.8)$ & $0(0)$ & 0.232 \\
\hline Oral ulcers & $7(15.9)$ & $4(20)$ & 0.688 \\
\hline Arthritis & $41(93.2)$ & $14(70)$ & 0.013 \\
\hline Serositis & $2(40 \%)$ & $12(60)$ & 0.112 \\
\hline Hematologic involvement & $27(61.4)$ & $14(70)$ & 0.504 \\
\hline Renal involvement & $18(40.9)$ & $7(35)$ & 0.635 \\
\hline Neuropsychiatric involvement & $8(18.2)$ & $4(20)$ & 0.863 \\
\hline ANA & $44(100)$ & $19(90.4)$ & 0.189 \\
\hline Anti-dsDNA, anti-Sm, or antiphospholipid & $25(56.8)$ & $11(57.9)$ & 0.937 \\
\hline Raynaud's phenomenon, n (\%) & $25(56.8)$ & $17(81)$ & 0.057 \\
\hline Livedo reticularis, n (\%) & $0(0)$ & $1(4.8)$ & 0.145 \\
\hline Arterial thrombosis, n (\%) & $0(0)$ & $1(4.8)$ & 0.145 \\
\hline Venous thrombosis, n (\%) & $7(15.9)$ & $5(23.8)$ & 0.443 \\
\hline SLEDAI & $1.91 \pm 3.16$ & $2.56 \pm 3.79$ & 0.559 \\
\hline Pregnancy, n (\%) & $26(59.1)$ & $14(66.7)$ & 0.557 \\
\hline Miscarriage, n (\%) & $4(9.1)$ & $6(28.6)$ & 0.042 \\
\hline Anti-SM, n (\%) & $10(22.7)$ & $4(23.5)$ & 0.947 \\
\hline Anti-RNP, n (\%) & $19(43.2)$ & $11(64.7)$ & 0.132 \\
\hline Anti-Ro, n (\%) & $11(25)$ & $5(29.4)$ & 0.725 \\
\hline Anti-La, n (\%) & $5(11.4)$ & $2(11.8)$ & 0.965 \\
\hline Positive anti- aCL IgM, n (\%) & $3(7)$ & $2(11.1)$ & 0.591 \\
\hline Positive anti- aCL IgG, n (\%) & $7(16.3)$ & $3(16.7)$ & 0.970 \\
\hline Positive anti-dsDNA, n (\%) & $15(34.1)$ & $3(17.6)$ & 0.207 \\
\hline $\mathrm{C} 2(\mathrm{mg} / \mathrm{dL})$ & $91.4 \pm 25.7$ & $86.9 \pm 29.8$ & 0.690 \\
\hline $\mathrm{CH} 100(\mathrm{mg} / \mathrm{dL})$ & $91.5 \pm 24.8$ & $89.4 \pm 30.2$ & 0.957 \\
\hline Hemoglobin (g/dL) & $12.5 \pm 1.3$ & $13.2 \pm 1.5$ & 0.138 \\
\hline Leukocytes $\left(/ \mathrm{mm}^{3}\right)$ & $6.317 \pm 3.120$ & $6.149 \pm 3.766$ & 0.977 \\
\hline Lymphocytes $\left(/ \mathrm{mm}^{3}\right)$ & $1.643 \pm 899$ & $1.477 \pm 675$ & 0.617 \\
\hline Platelets $\left(/ \mathrm{mm}^{3}\right)$ & $251.295 \pm 101.691$ & $197.500 \pm 68.534$ & 0.028 \\
\hline Creatinine (mg/dL) & $0.73 \pm 0.23$ & $0.86 \pm 0.36$ & 0.077 \\
\hline CRP $(\mathrm{mg} / \mathrm{dL})$ & $9.02 \pm 14.3$ & $12.10 \pm 37.32$ & 0.314 \\
\hline 24-h urine protein $(\mathrm{mg} / \mathrm{kg} / 24 \mathrm{~h})$ & $0.36 \pm 0.52$ & $0.26 \pm 0.25$ & 0.785 \\
\hline
\end{tabular}

Data are expressed as the mean \pm standard deviation or $\mathrm{n}(\%)$. ACR American College of Rheumatology, ANA antinuclear antibodies, anti-aCL anticardiolipin, CRP Creactive protein, $\cap P A H$ no PAH, PAH pulmonary arterial hypertension, SLE systemic lupus erythematosus, SLEDAl Systemic Lupus Erythematosus Disease Activity Index 
Table 2 Abnormalities on nailfold capillaroscopy among patients with SLE associated with PAH (SLE-PAH) or not associated with PAH (SLE-nPAH)

\begin{tabular}{llll}
\hline & SLE-PAH $n=16$ & SLE-nPAH $n=44$ & \\
\hline Capillaroscopy pattern & & & $7(15.9)$ \\
$\quad$ SD, $n(\%)$ & $9(56.3)$ & $37(84.1)$ & 0.002 \\
$\quad$ Normal or unspecific, $n(\%)$ & $7(43.7)$ & $9.18 \pm 1.77$ & 0.081 \\
Number of loops/mm & $8.63 \pm 1.28$ & $0.3 \pm 0.52$ & 0.134 \\
Microhemorrhages & $1.03 \pm 1.67$ & $0.85 \pm 1.72$ & 0.027 \\
Dilated capillaries & $1.39 \pm 1.46$ & $0.03 \pm 0.44$ & 0.468 \\
Giant capillaries & $0.04 \pm 0.11$ & $0.12 \pm 0.28$ & 0.010 \\
Avascular score & $0.45 \pm 0.63$ &
\end{tabular}

Data are expressed as the mean \pm standard deviation or $n$ (\%). NC nailfold capillaroscopy, $n P A H$ no PAH, PAH pulmonary arterial hypertension, SD scleroderma pattern, SLE systemic lupus erythematosus

area was $26.2 \pm 5.5 \mathrm{~cm}^{2}$. Pericardial effusion was detected in $9.5 \%$ of the patients.

Among the medications for the treatment of SLE, current or previous use of corticosteroids and antimalarial agents was significantly more frequent in the SLE-nPAH group than in the SLE-PAH group (100\% versus $85 \%$, respectively, for both medications; $p=$ 0.009). Mycophenolate and cyclophosphamide was also significantly more frequently used in the SLE-nPAH group than in the SLE-PAH group $(29.5 \%$ versus $5 \%$, respectively, $p=0.028 ; 56.8 \%$ versus $15 \%$, respectively, $p=0.002$ ) (Table 4). A higher proportion of patients used contraceptives in the SLE-PAH group than in the SLE-nPAH group $(52.6 \%$ versus $22.7 \%$, respectively; $p=0.019)$. Regarding the medications used for the treatment of PAH, $57.1 \%$ of the participants used sildenafil and $47.6 \%$ bosentan; five patients $(23.8 \%)$ received combined therapy. Ten patients $(47.6 \%)$ had received intravenous cyclophosphamide pulse therapy and eight (38.1\%) intravenous corticosteroids at the time of diagnosis of PAH (Table 4). Thirteen patients (61.9\%) had received oral anticoagulants. Six patients $(28.6 \%)$ in the SLE-PAH group died during follow-up.

As shown in Table 5, there were no significant differences in demographic, clinical, or laboratory variables between NYHA class I/II and NYHA class III/IV SLE-PAH patients. Although MPAP, CI, and PVRI were

Table 3 Results of right heart catheterization for patients with $\mathrm{PAH}$

\begin{tabular}{ll}
\hline & SLE-PAH $n=21$ \\
\hline $\operatorname{MPAP}(\mathrm{mm} \mathrm{Hg})$ & $48.9 \pm 11.7$ \\
$\mathrm{Cl}\left(\mathrm{L} / \mathrm{min} / \mathrm{m}^{2}\right)$ & $2.47 \pm 0.7$ \\
PCWP $(\mathrm{mm} \mathrm{Hg})$ & $10.7 \pm 2.5$ \\
PVRI $\left(\mathrm{WU} / \mathrm{m}^{2}\right)$ & $10.9 \pm 8.8$
\end{tabular}

Data are expressed as the mean \pm standard deviation. MPAP mean pulmonary artery pressure, $\mathrm{Cl}$ cardiac index, $P A H$ pulmonary arterial hypertension, $P C W P$ pulmonary capillary wedge pressure, $P V R I$ pulmonary vascular resistance index SLE systemic lupus erythematosus worse in class III/IV patients than in class I/II patients, these differences were not significant. There were 5 deaths in the NYHA class III and IV group and only one death in the NYHA class I and II group. No association was found between MPAP or CI values on RHC and clinical or laboratory variables (data not shown).

In the univariate analysis, miscarriage, RP, and SD pattern on capillaroscopy were significant risk factors for PAH ( $p=0.05 ; p=0.06 ; p=0.003$, respectively). In turn, arthritis was a protective factor against the development of PAH ( $p=0.05)$ (Table 6). In the multivariate analysis, SD pattern on NC was the single variable associated with an increased risk of PAH, with an OR of 6.393 (95\% CI: $1.530-26.716 ; p=0.011$ ).

\section{Discussion}

PAH is a devastating disorder that might lead to right ventricular dysfunction and, consequently, death. Recent studies have suggested that PAH associated with SLE involves heterogeneous conditions with a variable response to treatment. The identification of risk factors might contribute to improved screening and treatment management. To the best of our knowledge, this is the first study to analyze NC among patients with SLE associated with PAH. Thus, in the present study there was a 6.3-fold higher risk of PAH development in patients with the SD pattern on capillaroscopy.

$\mathrm{NC}$ is useful for analyses of microvascular abnormalities in the peripheral circulation and for the early diagnosis of SSc [28]. The SD pattern occurs in up to $98 \%$ of patients with SSc, and it may be found in 2 to $15 \%$ of patients with SLE $[28,31]$. In a study of SLE patients, the SD pattern was associated with the presence of RP and anti-RNP antibodies, which were associated with PAH in other studies [31].

$\mathrm{NC}$ abnormalities in SSc are associated with more severe visceral involvement and digital ulcers [21, 32]. Studies evaluating NC in patients with scleroderma and PAH found a reduced capillary density among patients 
Table 4 Treatment for SLE and PAH

\begin{tabular}{|c|c|c|c|}
\hline & SLE-PAH $n=21$ & SLE-nPAH $n=44$ & $P$-value \\
\hline Corticosteroids, n (\%) & $17(85)$ & $44(100)$ & 0.009 \\
\hline Antimalarial agents, n (\%) & $17(85)$ & $44(100)$ & 0.009 \\
\hline \multicolumn{4}{|l|}{ Immunosuppressants, n (\%) } \\
\hline Azathioprine & $8(40)$ & $25(56.8)$ & 0.212 \\
\hline Mycophenolate & $1(5)$ & $13(29.5)$ & 0.028 \\
\hline Cyclophosphamide & $3(15)$ & $25(56.8)$ & 0.002 \\
\hline Methotrexate & $11(55)$ & $19(43.2)$ & 0.380 \\
\hline \multicolumn{4}{|l|}{ PAH treatment } \\
\hline Sildenafil, n (\%) & $12(57.1)$ & - & \\
\hline Bosentan, n (\%) & $10(47.6)$ & - & \\
\hline Cyclophosphamide IV, n (\%) & $10(47.6)$ & - & \\
\hline Methylprednisolone IV, n (\%) & $8(38.1)$ & - & \\
\hline Oral anticoagulants, n (\%) & $13(65)$ & - & \\
\hline
\end{tabular}

$n P A H$ no $P A H, P A H$ pulmonary arterial hypertension, SLE systemic lupus erythematosus

with PAH compared with those without PH [22, 24]. In a study of 24 patients with SSc, 12 with and 12 without PAH, Riccieri et al., observed greater devascularization and a higher frequency of the active and late NC pattern compared with the early pattern among patients with $\mathrm{PAH}$ [23]. In addition, more severe $\mathrm{NC}$ abnormalities, such as a higher avascular score and lower capillary density, have been associated with a higher MPAP, suggesting an association between pulmonary arterial disease and the degree of abnormalities on NC $[22,23]$. Interestingly, Hofstee et al., observed an inverse correlation between pulmonary arterial pressure and capillary density among patients with PAH, either idiopathic or secondary to SSc [22].

In our study, in addition to the higher frequency of the SD pattern among patients with $\mathrm{PAH}$, we also found a higher degree of devascularization and larger number of dilated capillaries among patients with PAH compared with those without PAH. Although not fully elucidated, the pathogenesis of PAH involves an imbalance between vasodilation and vasoconstrictor mediators, and excessive vasoconstriction and increased PVR have been associated with endothelial and smooth muscle proliferation and pulmonary vascular remodeling $[16,18]$. The findings of the present study suggest that the peripheral microangiopathy might have a similar pathogenesis to pulmonary vascular bed microangiopathy. Our findings further suggest that patients with SSc and SLE share similar pathogenic mechanisms involved in the development of PAH.

The multivariate analysis did not reveal an association with variables previously reported as correlating with a higher risk of PAH, such as the presence of RP, serositis, and anti-RNP and anticardiolipin antibodies. This discrepancy might derive from the sample characteristics or the small number of patients with PAH analyzed.
Several studies have described an association of RP and digital vasculitis with $\mathrm{PAH}[19,33-36]$. In the present study, $81 \%$ of the patients with SLE and PAH and $56 \%$ of the patients without PAH exhibited RP. By univariate analysis, the presence of RP was associated with a 3.2-fold higher risk of PAH, which suggests that this variable might be relevant for the identification of this subpopulation of patients.

Anti-RNP and antiphospholipid antibodies have also been associated with an elevated risk of PAH among patients with SLE [19, 37-39]. Several autoantibodies might cause endothelial damage, vasoconstriction, and immunocomplex formation, and might be deposited on the pulmonary arterial wall [37]. In a study of Chinese individuals, anti-RNP and anticardiolipin antibodies were independent predictors of PAH among SLE patients, with ORs of 5.3 and 3.7, respectively [19]. A systematic review also of Chinese patients reported a higher frequency of anti-RNP (51.5\%) and anticardiolipin (46.6\%) antibodies among patients with SLE and PAH [38]. While some studies found a higher prevalence of anti-RNP antibodies among patients with SLE and $\mathrm{PAH}$, other did not find a significant difference between SLE patients with or without PAH $[11,36]$. In the present study, $65 \%$ of the patients with $\mathrm{PAH}$ had positive anti-RNP compared with $43 \%$ of the patients without PAH.

Antiphospholipid antibodies are classically associated with antiphospholipid syndrome and a higher risk of arterial or venous thrombosis and recurrent pregnancy loss. Cefle et al. (2011), found a higher frequency of antiphospholipid antibodies among SLE patients diagnosed with PAH on echocardiogram compared with patients without PAH [37]. However, other studies have failed to find such association [11, 33, 36, 40]. In the present 
Table 5 Demographic and clinical characteristics of patients with SLE and PAH by NYHA functional classes I/II and III/IV

\begin{tabular}{|c|c|c|c|}
\hline & NYHA class $I / \| n=9$ & NYHA class III/IV $n=12$ & $P$-value \\
\hline Female sex, n (\%) & $9(100)$ & $11(91.7)$ & 0.375 \\
\hline Age at SLE diagnosis & $26.7 \pm 10.9$ & $30.3 \pm 9.9$ & 0.219 \\
\hline Age at PAH diagnosis & $35.8 \pm 11.7$ & $35.3 \pm 12.3$ & 0.922 \\
\hline SLE length at PAH diagnosis & $9.1 \pm 7.5$ & $4.9 \pm 4.9$ & 0.138 \\
\hline Smoking, n (\%) & $1(11)$ & $0(0)$ & 0.352 \\
\hline Miscarriage, n (\%) & $3(33)$ & $3(25)$ & 0.676 \\
\hline Pregnancy, n (\%) & $6(66.7)$ & $8(66.7)$ & 1.000 \\
\hline Raynaud's phenomenon, n (\%) & $7(77.8)$ & $10(81)$ & 0.748 \\
\hline Livedo reticularis, n (\%) & $1(11.1)$ & $0(0)$ & 0.237 \\
\hline Arterial thrombosis, n (\%) & $0(0)$ & $1(8.3)$ & 0.375 \\
\hline Venous thrombosis, n (\%) & $4(44.4)$ & $1(8.3)$ & 0.055 \\
\hline Anti-SM, n (\%) & $2(25)$ & $2(22.2)$ & 0.893 \\
\hline Anti-RNP, n (\%) & $4(50)$ & $7(77.8)$ & 0.232 \\
\hline Anti-Ro, n (\%) & $2(25)$ & $3(33.3)$ & 0.707 \\
\hline Anti-La, n (\%) & $0(0)$ & $2(22.2)$ & 0.156 \\
\hline Positive anti- aCL IgM, n (\%) & $1(12.5)$ & $1(10)$ & 0.867 \\
\hline Positive anti- aCL IgG, n (\%) & $1(12.5)$ & $2(20)$ & 0.671 \\
\hline Positive anti-dsDNA, n (\%) & $2(25)$ & $1(11.1)$ & 0.453 \\
\hline $\mathrm{C} 2(\mathrm{mg} / \mathrm{dL})$ & $85.2 \pm 34.3$ & $88.1 \pm 28.6$ & 0.859 \\
\hline $\mathrm{CH} 100(\mathrm{mg} / \mathrm{dL})$ & $83.8 \pm 30.2$ & $93.1 \pm 31.4$ & 0.579 \\
\hline CRP $(\mathrm{mg} / \mathrm{dL})$ & $3.43 \pm 2.01$ & $19.20 \pm 50.20$ & 1.000 \\
\hline SLEDAI & $2 \pm 4.5$ & $3.0 \pm 3.4$ & 0.299 \\
\hline 6-min walk test & $413 \pm 14.8$ & $417.7 \pm 92.9$ & 0.934 \\
\hline FVC (\%) & $84.1 \pm 17.5$ & $75.7 \pm 13.2$ & 0.143 \\
\hline Dco (\%) & $52.2 \pm 8.3$ & $44.5 \pm 23.2$ & 0.548 \\
\hline Death, n (\%) & $1(11.1)$ & $5(41.7)$ & 0.125 \\
\hline \multicolumn{4}{|l|}{ Nailfold capillaroscopy } \\
\hline SD pattern, n (\%) & $4(66.7)$ & $5(50)$ & \multirow[t]{2}{*}{0.515} \\
\hline Normal or unspecific & $2(33.3)$ & $5(50)$ & \\
\hline \multicolumn{4}{|l|}{$\mathrm{RHC}$} \\
\hline MPAP & $47.6 \pm 7.7$ & $50.0 \pm 14.6$ & 0.654 \\
\hline $\mathrm{Cl}$ & $2.61 \pm 0.66$ & $2.36 \pm 0.75$ & 0.441 \\
\hline PVRI & $8.86 \pm 3.0$ & $12.62 \pm 11.47$ & 0.824 \\
\hline \multicolumn{4}{|l|}{ Treatment } \\
\hline Sildenafil, n (\%) & $4(44.4)$ & $8(67)$ & 0.309 \\
\hline Bosentan, n (\%) & $5(55.6)$ & $5(41.7)$ & 0.528 \\
\hline Cyclophosphamide IV, n (\%) & $4(44.4)$ & $6(50)$ & 0.801 \\
\hline Methylprednisolone IV, n (\%) & $3(33.3)$ & $5(41.7)$ & 0.697 \\
\hline Oral anticoagulants, n (\%) & $6(66.7)$ & $7(63.6)$ & 0.642 \\
\hline
\end{tabular}

Data are expressed as the mean \pm standard deviation or $\mathrm{n}(\%)$. SLE systemic lupus erythematosus, PAH pulmonary arterial hypertension, anti-aCL anticardiolipin, $\mathrm{Cl}$ cardiac index, CRP C-reactive protein, Dco carbon monoxide diffusing capacity, FVC forced vital capacity, IV intravenous, MPAP mean pulmonary artery pressure, NYHA New York Heart Association, PVRI pulmonary vascular resistance index, SLE systemic lupus erythematosus, SLEDAI Systemic Lupus Erythematosus Disease Activity Index 
Table 6 Variables associated with PAH among SLE patients in the univariate logistic regression analysis

\begin{tabular}{|c|c|c|c|}
\hline Variables & Odds ratio & $95 \% \mathrm{Cl}$ & $P$-value \\
\hline Sex & 0.952 & $0.081-11.134$ & 0.969 \\
\hline Age at SLE diagnosis & 1.019 & $0.972-1.069$ & 0.437 \\
\hline Pregnancy & 1.385 & $0.466-4.111$ & 0.558 \\
\hline Miscarriage & 4.000 & $0.989-16.179$ & 0.052 \\
\hline Smoking & 0.526 & $0.055-5.035$ & 0.577 \\
\hline Raynaud's phenomenon & 3.230 & $0.933-11.182$ & 0.064 \\
\hline Venous thrombosis & 1.652 & $0.455-5.993$ & 0.445 \\
\hline SLEDAI (total) & 0.945 & $0.802-1.113$ & 0.501 \\
\hline Malar rash & 0.812 & $0.141-4.640$ & 0.809 \\
\hline Photosensitivity & 0.313 & $0.057-1.703$ & 0.179 \\
\hline Arthritis & 0.136 & $0.018-1.003$ & 0.050 \\
\hline Serositis & 3.172 & $0.708-14.213$ & 0.131 \\
\hline Renal involvement & 0.881 & $0.191-4.068$ & 0.871 \\
\hline Neuropsychiatric involvement & 0.560 & $0.105-2.989$ & 0.498 \\
\hline Anti-Sm & 1.046 & $0.278-3.932$ & 0.947 \\
\hline Anti-RNP & 2.412 & $0.756-7.694$ & 0.137 \\
\hline Anti-La & 1.040 & $0.182-5.953$ & 0.965 \\
\hline Anti-Ro & 1.250 & $0.359-4.348$ & 0.726 \\
\hline Anticardiolipin IgM & 1.667 & $0.254-10.931$ & 0.594 \\
\hline Anticardiolipin lgG & 1.029 & $0.234-4.521$ & 0.970 \\
\hline Anti-DNA & 0.414 & $0.103-1.670$ & 0.215 \\
\hline $\mathrm{C} 2$ & 1.006 & $0.984-1.029$ & 0.574 \\
\hline $\mathrm{CH} 100$ & 1.003 & $0.981-1.026$ & 0.787 \\
\hline CRP & 1.005 & $0.984-1.027$ & 0.634 \\
\hline SD pattern on capillaroscopy & 6.796 & $1.897-24.345$ & 0.003 \\
\hline
\end{tabular}

95\% Cl 95\% confidence interval, CRP C-reactive protein, $P A H$ pulmonary arterial hypertension, SD scleroderma pattern, SLE systemic lupus erythematosus

study, we did not observe a significant difference in the proportion of aCL IgM and IgG between the groups. One should note that the measurement of lupus anticoagulants and anti-beta-2 glycoprotein I is not available at our service, and thus the levels of antiphospholipid antibodies might have been underestimated.

In agreement with previous studies, we did not find an association between disease activity as assessed by SLEDAI and the development of PAH [34, 39, 41].

Miscarriage occurred in a significantly higher proportion of patients in the SLE-PAH group, all of which occurred prior to the diagnosis of PAH. It is noteworthy that termination of pregnancy should be considered for patients with PAH due to the high risk of maternal death [42].

NYHA functional class, 6MWT, and hemodynamic parameters have considerable clinical relevance, as they are the main final outcomes in PAH and have strong association with mortality [3]. Upon investigating possible correlations between the severity of PAH and clinical and laboratory markers, we did not find a difference between patients with a higher (NYHA class III/IV) or lower (NYHA class I/II) functional class severity. In addition, we did not identify an association between $\mathrm{CI}$ and MPAP in the RHC with the analyzed variables. However, five deaths occurred among the NYHA class III/IV patients versus only one among the NYHA class I/II patients, indicating more severe disease among patients with higher functional class.

The treatment for PAH has undergone substantial changes over the past decades. Pulmonary vasodilators, such as prostacyclin analogs, endothelin receptor antagonists, and phosphodiesterase- 5 inhibitors, have significantly improved symptoms and reduced the rate of clinical deterioration [3]. In the present study, $57 \%$ of the patients had received sildenafil, $47 \%$ bosentan, and $23.8 \%$ combined treatment; none of the patients had received prostacyclin analogs, which remain scarcely available in our country. Because the immune system and/or inflammatory response abnormalities seem to be involved in the pathogenesis of $\mathrm{PAH}$, especially among patients with SLE, immunosuppressant therapy is suggested for patients with SLE and PAH [42-45]. In our study, $47 \%$ of the patients had received cyclophosphamide pulse therapy for PAH.

The present study has some limitations, such as the small number of patients with $\mathrm{PAH}$ and prior exposure of some patients in the control group to NC, which might have resulted in selection bias, i.e., the inclusion of a larger number of patients with RP. A further limitation derives from the retrospective design of the study, which was based on a review of medical records, hindering the analysis of relevant aspects such as the response to PAH treatment. In addition, only a portion of the patients with SLE but without PAH (43\%) underwent an echocardiogram for PAH screening, and thus, we could not rule out the possible inclusion of some asymptomatic PAH patients in the normal control group. Although we excluded patients with SSc overlap syndrome, we could not rule out the possible inclusion of patients with subclinical SSc in the study.

It is worth noting that this study included only patients with PAH confirmed on RHC, which is considered the gold standard for both the diagnosis and analysis of factors related to a poorer prognosis, such as elevated right atrial pressure and reduced CI [2, 3]. Due to its low prevalence, there is no recommendation for screening patients with SLE for PAH, in contrast to patients with SSc. Nevertheless, Khanna et al. (2013) [45], recently suggested that screening with Doppler echocardiography, pulmonary function tests with Dco, and serum markers, such as $\mathrm{N}$-terminal pro-B-type natriuretic peptide (NT-proBNP), should be performed for patients with mixed connective tissue disease or SLE with SSc-related manifestations and symptoms suggestive of PAH. 


\section{Conclusions}

In conclusion, the frequency of the SD pattern on $\mathrm{NC}$ was significantly increased among SLE patients with $\mathrm{PAH}$ compared with those without PAH. The presence of the SD pattern was associated with a 6.7 times higher risk of PAH. Thus, the present results lead us to consider the relevance of capillaroscopy evaluation for SLE patients, who are also at high risk of developing this serious and potentially fatal complication. We suggest the utilization of $\mathrm{NC}$, a non-invasive method, as a screening method for patients with SLE and further annual PH screening for patients who present the SD pattern, as currently conducted for patients with SSc. Prospective and multicenter studies are needed to better elucidate the role of $\mathrm{NC}$ in the determination of the risk of PAH development among patients with SLE, as well as to confirm the present results.

\section{Acknowledgements}

Not applicable.

\section{Funding}

No funding was received.

\section{Availability of data and materials}

The data used and analyzed during the current study are available from the corresponding author on reasonable request.

\section{Authors' contributions}

JFSD, EVMF, JOA, and CK contributed to the conception and design of the study, and to the acquisition of the data. JFSD and CK contributed to analysis and interpretations of data, and were the major contributor in writing the manuscript. All authors read and approved the final manuscript.

\section{Ethics approval and consent to participate}

The study was approved by the local research ethics committee at the Federal University of São Paulo (no. 503,630). All patients gave their informed consent prior to their inclusion in the study.

\section{Consent for publication}

Not applicable.

\section{Competing interests}

The authors declare that they have no competing interests.

\section{Publisher's Note}

Springer Nature remains neutral with regard to jurisdictional claims in published maps and institutional affiliations.

\section{Author details}

${ }^{1}$ Rheumatology Division, Medicine Department, Escola Paulista de Medicina, Universidade Federal de São Paulo (UNIFESP), São Paulo, SP, Brazil. ²Division of Pneumology, Medicine Department, Escola Paulista de Medicina, Universidade Federal de São Paulo (UNIFESP), São Paulo, SP, Brazil. ${ }^{3}$ Disciplina de Reumatologia da Universidade Federal de São Paulo, Rua Botucatu 740, 3 ` andar, São Paulo, SP 04023-062, Brazil.

Received: 20 November 2018 Accepted: 20 December 2018 Published online: 06 January 2019

\section{References}

1. Tsokos GC. Sytemic lupus erythematosus. N Engl J Med. 2011;365:2110-21.

2. Hoeper MM, Bogaard HJ, Condliffe R, Frantz R, KhannaD KM, et al. Definitions and diagnosis of pulmonary hypertension. J Am Coll Cardiol. 2013;(62 Suppl):D42-50.
3. Galiè N, Humbert M, Vachiery JL, Gibbs S, Lang I, Torbicki A, et al. 2015 ESC/ ERS Guidelines for the diagnosis and treatment of pulmonary hypertension. Eur Heart J. 2016;37:67-119.

4. Galiè N, Manes A, Farahani KV, Pelino F, Palazzini M, Negro L, et al. Pulmonary arterial hypertension associated to connective tissue diseases. Lupus. 2005;14:713-7.

5. McGoon MD, Miller DP. REVEAL: a contemporary US pulmonary arterial hypertension registry. Eur Respir Rev. 2012;21(123):8-18.

6. Peacock AJ, Murphy NF, McMurray JJV, Caballero L, Stewart S. An epidemiological study of pulmonary arterial hypertension. Eur Respir J. 2007;30:104-9.

7. Johnson SR, Granton JT. Pulmonary hypertension in systemic sclerosis and systemic lupus erythematosus. Eur Respir Rev. 2011;20:277-86.

8. Chung L, Liu J, Parsons L, Hassoun PM, McGoon M, Badesch DB, et al. Characterization of connective tissue disease-associated pulmonary arterial hypertension from REVEAL: identifying systemic sclerosis as a unique phenotype. Chest. 2010;138:1383-94.

9. Haas C. Pulmonary hypertension associated with systemic lupus erythematosus. Bull Acad Natl Med. 2004;188:985-97.

10. Pope J. An update in pulmonary hypertension in systemic lupus erythematosus - do we need to know about it? Lupus. 2008;17:274-7.

11. Prabu A, Patel K, Yee CS, Nightingale P, Situnayake RD, Thickett DR, et al. Prevalence and risk factors of pulmonary arterial hypertension in patients with lupus. Rheumatology. 2009;48:1506-11.

12. Simonson JS, Schiller NB, Petri M, Hellman DB. Pulmonary hypertension in systemic lupus erythematosus. J Rheumatol. 1989;16:918-25.

13. Foïs E, Le Guern V, Dupuy A, Humbert M, Mouthon L, Guillevin L. Noninvasive assessment of systolic pulmonary artery pressure in systemic lupus erythematosus: retrospective analysis of 93 patients. Clin Exp Rheumatol. 2010;28:836-41.

14. Chow SL, Chandran V, Fazelzad R, Johnson SR. Prognostic factors for survival in systemic lupus erythematosus associated pulmonary hypertension. Lupus. 2012;21:353-64.

15. Qian J, Wang Y, Huang C, Yang X, Zhao J, Wang Q, et al. Survival and prognostic factors of systemic lupus erythematosus-associated pulmonary arterial hypertension: a PRISMA-compliant systematic review and metaanalysis. Autoimmun Rev. 2016;15:250-7.

16. Tselios K, Gladman DD, Urowitz MB. Systemic lupus erythematosus and pulmonary arterial hypertension: links, risks, and management strategies. Open Access Rheumatol. 2016;9:1-9.

17. Condliffe R, Kiely DG, Peacock AJ, Corris PA, Gibbs JS, Vrapi F, et al. Connective tissue disease-associated pulmonary arterial hypertension in the modern treatment era. Am J Respir Crit Care Med. 2009;179:151-7.

18. Dhala A. Pulmonary arterial hypertension in systemic lupus erythematosus: current status and future direction. Clin Dev Immunol 2012;2012:854-941.

19. Lian F, Chen D, Wang Y, Ye Y, Wang $X$, Zhan Z, et al. Clinical features and independent predictors of pulmonary arterial hypertension in systemic lupus erythematosus. Rheumatol Int. 2012;32:1727-31.

20. Huang C, Li M, Liu Y, Wang Q, Guo X, Zhao J, et al. Baseline characteristics and risk factors of pulmonary arterial hypertension in systemic lupus erythematosus patients. Medicine (Baltimore). 2016;95:e2761.

21. Souza EJ, Kayser C. Nailfold capillaroscopy: relevance to the practice of rheumatology. Rev Bras Reumatol. 2015;55:264-71.

22. Hofstee HM, Vonk Noordegraaf A, Voskuyl AE, Dijkmans BA, Postmus PE, Smulders YM, et al. Nailfold capillary density is associated with the presence and severity of pulmonary arterial hypertension in systemic sclerosis. Ann Rheum Dis. 2009;68:191-5

23. Riccieri V, Vasile M, lannace $N$, Stefanantoni $K$, Sciarra I, Vizza CD, et al. Systemic sclerosis patients with and without pulmonary arterial hypertension: a nailfold capillaroscopy study. Rheumatology. 2013;52:1525-8.

24. Ong YY, Nikoloutsopoulos T, Bond CP, Smith MD, Ahern MJ, RobertsThomson PJ. Decreased nailfold capillary density in limited scleroderma with pulmonary hypertension. Asian Pac J Allergy Immunol. 1998;16:81-6.

25. Ohtsuka T, Hasegawa A, Nakano A, Yamakage A, Yamaguchi M, Miyachi Y. Nailfold capillary abnormality and pulmonary hypertension in systemic sclerosis. Int J Dermatol. 1997;36:116-22.

26. Hochberg MC. Updating the American College of Rheumatology revised criteria for the classification of systemic lupus erythematosus. Arthritis Rheum. 1997:40:1725.

27. Bombardier C, Gladman DD, Urowitz MB, Caron D, Chang CH. Derivation of the SLEDAI. A disease activity index for lupus patients. The committee on prognosis studies in SLE. Arthritis Rheum. 1992;35:630-40. 
28. Maricq HR, LeRoy EC, D'Angelo WA, Medsger TA Jr, Rodnan GP, Sharp GC, et al. Diagnostic potential of in vivo capillary microscopy in scleroderma and related disorders. Arthritis Rheum. 1980;23:183-8.

29. Sekiyama JY, Camargo CZ, Andrade LE, Kayser C. Reliability of Widefield Nailfold Capillaroscopy and Videocapillaroscopy in the assessment of patients with Raynaud's Phenomenon. Arthritis Care Res. 2013;65:1853-61.

30. The Criteria Committee of the New York Heart Association. Nomenclature and criteria for diagnosis of diseases of the heart and great vessels. 9th ed. Boston: Lippincott Williams and Wilkins; 1994.

31. Furtado RN, Pucinelli ML, Cristo W, Andrade LE, Sato El. Scleroderma-like nailfold capillaroscopic abnormalities are associated with anti-U1-RNP antibodies and Raynaud's phenomenon in SLE patients. Lupus. 2002;11:3541.

32. Herrick AL, Moore TL, Murray AK, Whidby N, Manning JB, Bhushan M, et al. Nail-fold capillary abnormalities are associated with anti-centromere antibody and severity of digital ischemia. Rheumatology. 2010;49:1776-82.

33. Li EK, Tam LS. Pulmonary hypertension in systemic lupus erythematosus: clinical association and survival in 18 patients. J Rheumatol. 1999;26:1923-9.

34. Asherson RA, Higenbottam TW, Dinh Xuan AT, Khamashta MA, Hughes GR. Pulmonary hypertension in a lupus clinic: experience with twenty-four patients. J Rheumatol. 1990;17:1292-8.

35. Kasparian A, Floros A, Gialafos E, Kanakis M, Tassiopoulos S, Kafasi N, et al. Raynaud's phenomenon is correlated with elevated systolic pulmonary arterial pressure in patients with systemic lupus erythematosus. Lupus. 2007; 16:505-8.

36. Min HK, Lee JH, Jung SM, Lee J, Kang KY, Kwok SK, et al. Pulmonary hypertension in systemic lupus erythematosus: an independent predictor of patient survival. Korean J Intern Med. 2015;30:232-41.

37. Cefle A, Inanc M, Sayarlioglu M, Kamali S, Gul A, Ocal L, et al. Pulmonary hypertension in systemic lupus erythematosus: relationship with antiphospholipid antibodies and severe disease outcome. Rheumatol Int. 2011;31:183-9.

38. Xia YK, Tu SH, Hu YH, Wang Y, Chen Z, Day HT, et al. Pulmonary hypertension in systemic lupus erythematosus: a systematic review and analysis of 642 cases in Chinese population. Rheumatol Int. 2013;33:1211-7.

39. Kamel SR, Omar GM, Darwish AF, Asklany HT, Ellabban AS. Asymptomatic pulmonary hypertension in systemic lupus erythematosus. Clin Med Insights Arthritis Musculoskelet Disord. 2011;4:77-86.

40. Petri M, Rheinschmidt M, Whiting-O'Keefe Q, Hellmann D, Corash L. The frequency of lupus anticoagulant in systemic lupus erythematosus. A study of sixty consecutive patients by activated partial thromboplastin time, Russell viper venom time, and anticardiolipin antibody level. Ann Intern Med. 1987;106:524-31.

41. Chung SM, Lee CK, Lee EY, Yoo B, Lee SD, Moon HB. Clinical aspects of pulmonary hypertension in patients with systemic lupus erythematosus and in patients with idiopathic pulmonary arterial hypertension. Clin Rheumatol. 2006;25:866-72.

42. Galiè N, Hoeper MM, Humbert M, Torbicki A, Vachiery JL, Barbera JA, et al. Guidelines for the diagnosis and treatment of pulmonary hypertension: the Task Force for the Diagnosis and Treatment of Pulmonary Hypertension of the European Society of Cardiology (ESC) and the European Respiratory Society (ERS), endorsed by the International Society of Heart and Lung Transplantation (ISHLT). Eur Heart J. 2009;30:2493-537.

43. Tanaka E, Harigai M, Tanaka M, Kawaguchi Y, Hara M, Kamatani N. Pulmonary hypertension in systemic lupus erythematosus: evaluation of clinical characteristics and response to immunosuppressive treatment. J Rheumatol. 2002;29:282-7.

44. Sanchez O, Sitbon O, Jais X, Simonneau G, Humbert M. Immunosuppressive therapy in connective tissue diseases-associated pulmonary arterial hypertension. Chest. 2006;130:182-9.

45. Khanna D, Gladue H, Channick R, Chung L, Distler O, Furst DE, et al. Recommendations for screening and detection of connective tissue disease-associated pulmonary arterial hypertension. Arthritis Rheum. 2013;65:3194-201.

Ready to submit your research? Choose BMC and benefit from:

- fast, convenient online submission

- thorough peer review by experienced researchers in your field

- rapid publication on acceptance

- support for research data, including large and complex data types

- gold Open Access which fosters wider collaboration and increased citations

- maximum visibility for your research: over $100 \mathrm{M}$ website views per year

At $\mathrm{BMC}$, research is always in progress.

Learn more biomedcentral.com/submissions 